\title{
Development of a patient reported outcome scale for fatigue in multiple sclerosis: The Neurological Fatigue Index (NFI-MS)
}

\author{
Roger J Mills ${ }^{1 *}$, Carolyn A Young ${ }^{1}$, Julie F Pallant ${ }^{2}$, Alan Tennant ${ }^{3}$
}

\begin{abstract}
Background: Fatigue is a common and debilitating symptom in multiple sclerosis (MS). Best-practice guidelines suggest that health services should repeatedly assess fatigue in persons with MS. Several fatigue scales are available but concern has been expressed about their validity. The objective of this study was to examine the reliability and validity of a new scale for MS fatigue, the Neurological Fatigue Index (NFI-MS).
\end{abstract}

Methods: Qualitative analysis of 40 MS patient interviews had previously contributed to a coherent definition of fatigue, and a potential 52 item set representing the salient themes. A draft questionnaire was mailed out to 1223 people with MS, and the resulting data subjected to both factor and Rasch analysis.

Results: Data from 635 (51.9\% response) respondents were split randomly into an 'evaluation' and 'validation' sample. Exploratory factor analysis identified four potential subscales: 'physical', 'cognitive', 'relief by diurnal sleep or rest' and 'abnormal nocturnal sleep and sleepiness'. Rasch analysis led to further item reduction and the generation of a Summary scale comprising items from the Physical and Cognitive subscales. The scales were shown to fit Rasch model expectations, across both the evaluation and validation samples.

Conclusion: A simple 10-item Summary scale, together with scales measuring the physical and cognitive components of fatigue, were validated for MS fatigue.

\section{Background}

One of the symptoms causing the greatest morbidity and disability in multiple sclerosis (MS) is fatigue [1,2]. It has been suggested that health services should apply a broad range of approaches and repeatedly assess fatigue in persons with MS, to provide preventive care and appropriate interventions [3]. However, assessing fatigue is not easy since the symptom is inherently complex and the pathophysiology is not well explained [4,5]. A major problem has been the absence of a clear definition of fatigue [5-7] and, consequently, there is debate regarding the possible dimensionality of the phenomenon, with some arguing that fatigue can only be understood as a multidimensional entity,[8] while others argue that it is unidimensional [9]. This immediately poses a problem for quantification of fatigue, since an

\footnotetext{
* Correspondence: rjm@crazydiamond.co.uk

${ }^{1}$ The Walton Centre for Neurology and Neurosurgery, Liverpool, L9 7LJ, UK
}

unambiguous definition and unidimensionality are fundamental requirements of measurement.

Regardless of these issues, several scales to measure fatigue have been developed. For example, the Fatigue Severity Scale (FSS)[4] has been one of the most widely used fatigue scales for MS and, true to its origins, has often been employed to dichotomise groups into those with 'normal' levels of fatigue and those where fatigue had a disproportionately high impact. Another scale, the Modified Fatigue Impact Scale (MFIS)[10] has been recommended by the MS Council as an outcome measure for fatigue [5]. Despite their widespread use, some limitations have recently been observed with respect to these scales, suggesting that they do not satisfy modern standards of outcome measurement $[11,12]$. Such deficiencies suggest a need for a better definition of, and a high-quality measurement instrument for, fatigue [6]. Fatigue has been defined, as a result of qualitative analysis, as a: 
'reversible motor and cognitive impairment with reduced motivation, and a desire to rest, either appearing spontaneously or brought on separately by mental or physical activity, humidity, acute infection and food ingestion. It was relieved by daytime sleep or rest without sleep. It could occur at any time but was usually worse in the afternoon'[6].

In MS, fatigue could be daily, had usually been present for years and had greater severity than any pre-morbid fatigue. It was a synthesis of the features, which arose from that qualitative analysis, which defined the symptom and full details of this can be found elsewhere [6].

\section{Objective}

The current study takes this qualitative work forward to the next phase of measurement, with the aim of developing a valid and reliable patient reported outcome scale for fatigue, the Neurological Fatigue Index (NFIMS).

The items in the scale are based on the previous qualitative work. Table 1 provides some example of how the items relate to the thematic framework of the definition. The scale was developed to conform to Rasch measurement model standards,[13] and the U.S. Food and Drug Agency's (FDA) guidelines for the development of patient-reported outcome measures [14].

\section{Methods}

The study had approval from relevant local research ethics committees (Sefton EC115.03 and Hammersmith 05/Q0401/7). All subjects received written information on the study and gave written informed consent prior to participation.

\section{Sample and materials}

Initially, there were 57 potential items for the new scale each with a common four point, Likert-style response option [15] of 'strongly disagree', 'disagree', 'agree' and 'strongly agree', with each item being scored $0,1,2,3$. There was a single sentence instruction at the start of the scale asking respondents to consider their experience over the previous two weeks. Emphasis was placed on the dynamic quality or reversible nature of fatigue e.g., my limbs can become heavy rather than my limbs are heavy, in order that the scale should not be confounded by fixed neurological deficit. The nascent scale was put to an expert, multidisciplinary panel of ten professionals experienced in MS and fatigue, comprising: MS specialist nurses, MS specialist physiotherapists and occupational therapists, consultants in neurology and neurorehabilitation each with specialist interest in MS, a consultant rheumatologist and a clinical physiologist in sleep medicine, in order to confirm that items and their wording were reasonable.

The draft scale was subsequently administered, faceto-face, to 15 MS patients in the outpatient clinic. They were encouraged to give a running commentary during completion. This allowed identification and remedy of any gross problems with wording or item dysfunction. They were also asked to comment on the completeness of the item pool, and if any obvious features had been omitted.

A random cross-sectional cohort of 1223 patients with clinically definite MS,[16] identified from research databases in two centres in the UK (WCNN, Liverpool and Imperial College Healthcare Trust, London) was then sent packs, by mail, containing the set of potential items for the proposed scale, questions on demographics and basic disease information, together with other scales chosen for comparative analysis. Participants of any age, disease type, and disability level were included (the range of Expanded Disability Status Scale scores [17] (EDSS), was 0-9.0 as rated by neurologists at the time of database enrolment). Participants were also asked to estimate their best walking distance from a choice of four options, in order to corroborate EDSS at the time of questionnaire completion.

Table 1 Item origins

\begin{tabular}{lll}
\hline Framework & Feature & Item wording \\
\hline SE motor features & can develop weakness & Sometimes, I lose my body strength \\
SE cognitive features & concentrate on simple tasks & Sometimes, I really have to concentrate on what are usually simple things \\
SE motivation & thought puts off doing & The thought of having to do something often puts me off doing it \\
SE tiredness & tiredness & By the end of the day I'm shattered \\
Cadence & carry over & If I've overdone things, I know about it the next day \\
Precipitating/aggravating factors & physical exertion induces weakness & I soon become weak after physical effort \\
Relieving factors & day rest restorative & Resting allows me to carry on \\
Severity & weak at rest & I can become weak even if I've not been doing anything \\
Associated features & unrefreshing nocturnal sleep & When I awake in the morning, I feel unrefreshed \\
\hline
\end{tabular}

Examples of item wording representing the individual features of fatigue in the context of the thematic framework derived from the qualitative analysis. $\mathrm{SE}=$ subjective experience. 
The additional scales in the questionnaire pack were:

i) Visual analogue scale (VAS): a $10 \mathrm{~cm}$, modified (i.e. marked with $\mathrm{cm}$ gradations), horizontal visual analogue scale with anchors of 'lively and alert' (zero, left) and 'absolutely no energy to do anything at all' (10, right).

ii) Fatigue Severity Scale-5 (FSS-5): a short-form of the original nine item scale, including five items with a seven-point response option, modified from the original Rasch analysis in an MS population [11] iii) Modified Fatigue Impact Scale, Phys- 8 and Cog-5: an eight item physical scale and a five item cognitive fatigue scale modified from the original MFIS subscales by Rasch analysis in an MS population [12].

Retesting was performed at 2 to 4 weeks.

\section{Psychometric analysis/item reduction Initial exploration of dimensionality}

Given the multi-faceted nature of fatigue that had previously emerged from the qualitative analysis, and consistent with some of the published literature about the dimensionality of fatigue,[8] an exploratory factor analysis was undertaken to identify potential domains of fatigue. A Principal Components Analysis (PCA), based on a polychoric correlation matrix, was undertaken to extract the factors followed by oblique rotation of factors using Oblimin rotation (delta $=0$ ). Suitability of the data for factor analysis was tested by Bartlett's Test of Sphericity,[18] which should be significant, and the Kaiser-Meyer-Olkin (KMO) measure of sampling adequacy, which should be $>0.6[19,20]$. The number of factors to be retained was guided by three decision rules: Kaiser's criterion (eigenvalues above 1);[21] inspection of the screeplot,[22] and by the use of Horn's parallel analysis [23]. Parallel analysis is one of the most accurate approaches to estimating the number of components [24]. The size of eigenvalues obtained from PCA are compared with those obtained from a randomly generated data set of the same size. Only factors with eigenvalues exceeding the values obtained from the corresponding random data set are retained for further investigation. Parallel analysis was conducted using the software developed by Watkins [25].

Items identified to be associated in domains were taken forward to the Rasch analysis, to be analysed on a domain-specific basis and also to test if an overall summary scale could be derived.

\section{Rasch Analysis}

Rasch analysis is a modern psychometric approach which is widely used in the development, refinement and evaluation of patient reported outcome measures [13,26-28]. The Rasch model states that the probability of a person giving a certain answer to an item is a logistic function of the difference between the person's ability (in this case level of fatigue) and the item's difficulty (in this case the level of fatigue expressed by the item) [13]. Where the observed pattern of responses do not deviate too much from that expected by the model, the scale is said to satisfy Rasch model expectations. Full details of the process of Rasch analysis are given elsewhere $[29,30]$. Briefly, the process is concerned with whether or not the data meet the model expectations, and provides an assessment of the suitability of the response scale, the fit of individual items, item bias, and the dimensionality and targeting of the scale as a whole.

In summary, fit of data to the Rasch model was deemed acceptable if the following criteria were fulfilled:

1) ordered item category thresholds;

2) assumption of local independence holds (no significant $(>0.3)$ correlations in the residuals), reflecting that once account of the trait under consideration has been taken, the items do not display any further associations that would indicate redundancy or multidimensionality;

3) assumption of probabilistic ordering of items holds, determined by a range of fit statistics:

a. both total chi-square probability and individual item chi-square probability values non-significant (5\% alpha with Bonferroni correction for the number of items);

b. individual item fit residual, by convention, within $\pm 2.5(99 \% \mathrm{CI})$;

c. mean and SD of both summary item fit residual and person fit residuals approaching 0 and 1 respectively;

4) reliability (person-item separation index) greater than 0.85 ;

5) differential item functioning (DIF) absent for age, sex and disease duration as defined by a non-significant ANOVA (5\% alpha with Bonferroni correction). Where necessary, DIF was tested to see if it cancelled out at the test level [31]. In addition, DIF was used to test invariance of measurement across time in the test-retest analysis;

6) Strict unidimensionality assessed by comparing person estimates from two sets of items derived from the positive and negative loadings of the first component in PCA of the residuals. Unidimensionality is indicated if less than $5 \%$ of t-tests are significant (or the lower bound of the binomial confidence interval overlaps 5\%)[32,33].

The unrestricted (partial credit) Rasch polytomous model was used with a conditional pair-wise parameter estimation [34]. Failure of items to fit Rasch model expectations led to an iterative procedure using techniques for collapsing response categories, item deletion, and adjusting for DIF where necessary. 
For Rasch analysis, a sample size of 243 will provide accurate estimates of item and person locations irrespective of the scale targeting [35]. Assuming a 50\% response rate from the mail-out, that sample size would allow the data to be split randomly into two equal samples, one for the initial evaluation of the data set, the second to validate the results.

\section{External comparison}

Linear correlation of the Rasch derived interval level person estimates, from the new scale, was performed with the comparator measures, having also been transformed to interval scaling by Rasch analysis. Consequently, Pearson correlation coefficients were used between these estimates except for the VAS, which remained as an ordinal scale, and so Spearman correlation was used. All correlations were expected to be moderate (0.4-0.7) in size.

\section{Test-Retest Reliability}

The test-retest reliability of scales was undertaken with Spearman correlation on un-transformed data (to reflect how it is most likely to be used in a clinic setting). Values of $\geq 0.7$ are considered appropriate. In addition, median values are reported at both time points and their differences tested by a Wilcoxon Signed Rank test.

\section{Raw-Score to Interval scale conversion}

Given fit to the Rasch model, a straightforward conversion is available between the raw score for each scale, and the interval scale estimate provided by the model (the person location), in logits. The logit estimates are converted to the same range as the raw score by a further simple linear transformation. This nomogram can be used to obtain linear estimates from the raw scores of other samples only when their data are complete.

The Rasch analysis was performed using the RUMM 2020 software [36]. All other analysis was undertaken with SPSS version 15.

\section{Results}

\section{Review panel and cognitive debriefing}

All items were confirmed as being reasonable by the review panel; one additional item regarding morning sleep inertia was added. During the cognitive debriefing, six items were discarded because it was clear that they would not be relevant to all patients (e.g. reference to relapse and long journeys) and two items were reworded, producing a 52 item scale. Table 1 illustrates some of the pool items in the context of both the individual features of fatigue and the wider framework of the qualitative analysis.

\section{Sample characteristics}

635 packs were returned (635/1223, 51.9\% response). 451 (71\%) were female. Mean age was 46.6 years (SD 10.9, range $21-83), 54$ (8.5\%) had primary progressive disease, 337 (53.1\%) relapsing remitting and 177 (27.9\%) secondary progressive disease, 67 (10.6\%) had unknown disease type. The mean duration of MS was 15.1 years (SD 9.5, range 249). There was a wide range of EDSS scores (0-9.0).

\section{Psychometric analyses}

The main sample was split randomly into two, making an 'evaluation' and a 'validation' sample. Comparison of these samples by t-test or chi-square test across a range of characteristics revealed no significant differences (Table 2). A further 151 subjects completed the retest at 2-4 weeks.

\section{Factor analysis}

Bartlett's Test of Sphericity was highly significant ( $\mathrm{p}<$ 0.001 ) and the Kaiser-Meyer-Olkin (KMO) measure of sampling adequacy value of 0.94 , both supporting the factorability of the matrix. Principal Components Analysis with Oblimin rotation revealed four potential subscales from the 52 item set, which was also supported by parallel analysis. Thirty nine of the 52 items loaded substantially onto these four factors. After removing all items with standardised loadings of less than 0.4 , the resulting four factor solution, which explained $62 \%$ of the total variance, could be interpreted as representing physical (16 items); relief by diurnal sleep or rest (7 items); abnormal nocturnal sleep and sleepiness (8 items), and cognitive (8 items) (see Table 3).

\section{Rasch analysis}

Data in the evaluation sample for each of these domains were then fitted to the Rasch measurement model. An iterative process of item reduction involved identifying disordered thresholds, DIF, item misfit and breaches of local dependency, including multi-dimensionality. The summary findings related to the analysis of each domain are given in Table 4.

Physical scale Rasch analysis of the 16 Physical items identified in the PCA indicated that all item thresholds were ordered, suggesting respondents could properly discriminate between response options. There was no DIF by age, gender, or duration of disease. The 16 item set displayed multidimensionality (Table 4, analysis 1), with $14.6 \%$ (CI $12.2-17.0 \%$ ) of t-tests indicating significantly different person estimates derived from different subsets of items. An iterative process led to a scale reduction to 8 items. The resulting 8 item 'Physical' scale showed good fit to model expectations (Table 4, analysis 2) and just $4.13 \%$ of t-tests were significant, confirming a unidimensional scale.

Cognitive scale All thresholds were ordered and DIF was absent. Overall, the original 8 items failed to meet model expectations (Table 4, analysis 3). Two items showed local dependency: 'mental effort really takes it out of me' and 'Having to concentrate for too long makes me feel weak'. This meant that these items were very similar, more-or-less measuring the same thing, 
Table 2 Comparison of the evaluation and validation sample characteristics

\begin{tabular}{|c|c|c|c|c|}
\hline & Characteristic & Evaluation sample & Validation sample & $\begin{array}{l}\text { Difference between evaluation } \\
\text { and validating sample }\end{array}$ \\
\hline \multirow{8}{*}{ disease type, n (\%) } & number of subjects & 317 & 318 & \\
\hline & mean age (SD, min.-max.)(yrs) & $46.8(11.3)$ & $46.4(10.6)$ & t-test $p=0.606$ \\
\hline & number female (\%) & $234(73.8)$ & $217(68.2)$ & chi-square $p=0.144$ \\
\hline & mean disease duration (SD, min.-max.)(yrs) & $16.0(9.7,2-49)$ & $14.2(9.4,2-45)$ & t-test $p=0.064$ \\
\hline & $\mathrm{pp}$ & $25(7.9)$ & $29(9.1)$ & chi-square $p=0.932$ \\
\hline & rr & $169(53.3)$ & $168(52.8)$ & \\
\hline & $\mathrm{sp}$ & $88(27.8)$ & $89(28.0)$ & \\
\hline & unknown & $35(11.0)$ & $32(10.1)$ & \\
\hline \multirow[t]{6}{*}{ EDSS, n (\%) } & $0-4.0$ & $104(32.8)$ & $110(34.6)$ & chi-square $p=0.88$ \\
\hline & $4.5-6.5$ & $101(31.9)$ & $95(29.9)$ & \\
\hline & $7.0-7.5$ & $70(22.1)$ & $66(20.8)$ & \\
\hline & $8.0-9.5$ & $38(12.0)$ & $42(13.2)$ & \\
\hline & unknown & $4(1.3)$ & $5(1.6)$ & \\
\hline & mean 100 mm VAS fatigue score (SD, min.-max.) & $55.73(24.4,0-100)$ & $52.11(23.19,0-100)$ & t-test $p=0.059$ \\
\hline
\end{tabular}

$\mathrm{pp}=$ primary progressive, $\mathrm{rr}=$ relapsing remitting, $\mathrm{sp}=$ secondary progressive, $\mathrm{VAS}=$ visual analogue scale.

and so one would be redundant, After removal of misfitting items, a four item scale satisfied model expectations (Table 4, analysis 4) with strict unidimensionality.

Relief by diurnal sleep or rest scale The seven items from the diurnal sleep scale satisfied model expectations (Table 4, analysis 5). There was no local dependency, and the scale was strictly unidimensional. Two items showed DIF by gender: 'I need to rest in the day' and 'I try to rest or sleep beforehand, if I know I have to do something.... These were biased in opposite directions with males more likely to report a higher score on the former, and females the latter. At the scale level, the DIF cancelled out.

Abnormal nocturnal sleep and sleepiness scale All thresholds were ordered for the 8 item scale. One item, 'If I sleep in the day, I don't sleep well at night' displayed substantial misfit, and overall the scale failed to satisfy model expectations (Table 4, analysis 6). Removal of the misfitting item improved the overall fit of the scale, with no local dependency or DIF, and strict unidimensionality (Table 4, analysis 7).

Summary scale All items from the subscales above were then included as potential items for a summary scale (a higher order factor). This resulted in significant misfit to model expectations and a clear multidimensional structure (Table 4, analysis 8). The items split into two groups, a physical-cognitive component, and a sleep-rest component. From the former, a 10 item summary scale was derived, satisfying all aspects of model expectation (Table 4 , analysis 9). It was not possible to derive a summary scale for sleep, as the items consistently fractured into the two components of the diurnal and nocturnal sleep scales.

\section{Validation Data}

The data from the validation sample for each derived scale were then fitted to the Rasch model. The Physical, Cognitive, and Summary scales all demonstrated fit to model expectations, with ordered thresholds, no DIF for person factors, no local dependency and strict unidimensionality (Table 4, analyses 10-12). The two sleep scales required further modifications to adjust for misfit (nocturnal sleep) or multidimensionality (diurnal sleep) (Table 4, analyses 13 and 15). Satisfactory solutions were found for each scale (Table 4, analyses 14 and 16). There was no DIF by sample which further strengthened the validity of the fit across both the samples. The Physical, Cognitive, and Summary scales all achieved a level of reliability necessary for use in individuals.

\section{Targeting}

The final scales displayed acceptable person-item targeting with percentages of extreme scores of less than $5 \%$, apart from the cognitive scale which had a small ceiling effect of $7.2 \%$ and the physical scale which had a ceiling effect of $7.7 \%$ (Table 4, final column).

\section{Test-retest reliability}

Retesting was performed between 2 and 4 weeks. The invariance of the scales over time were confirmed by the absence of DIF. Test-retest reliability was good, with correlation coefficients above 0.7 at 2-4 weeks for all scales (Table 5). In addition, there were no significant differences in the median scores at the two time points (Wilcoxon Signed Rank; p > 0.05).

\section{External construct validity}

The correlations between the NFI-MS, and comparator measures, are shown in Table 6. Those correlations 
Table 3 Pattern matrix of four factor solution from PCA with Oblimin rotation

\begin{tabular}{|c|c|c|c|c|}
\hline & \multicolumn{4}{|c|}{ Component } \\
\hline Item & 1:Physical & 2: Cognitive & $\begin{array}{l}\text { 3: Diurnal } \\
\text { sleep/rest }\end{array}$ & $\begin{array}{l}\text { 4: Abnormal } \\
\text { sleep }\end{array}$ \\
\hline 19 & .783 & & & \\
\hline 01 & .739 & & & \\
\hline 09 & .736 & & & \\
\hline 51 & .736 & & & \\
\hline 22 & .733 & & & \\
\hline 03 & .732 & & & \\
\hline 10 & .730 & & & \\
\hline 11 & .718 & & & \\
\hline 20 & .706 & & & \\
\hline 18 & .702 & & & \\
\hline 27 & .697 & & & \\
\hline 12 & .686 & & & \\
\hline 21 & .658 & & & \\
\hline 02 & .610 & & & \\
\hline 28 & .541 & & & -.336 \\
\hline 26 & .529 & & & \\
\hline 29 & & -.842 & & \\
\hline 30 & & -.828 & & \\
\hline 17 & & -.783 & & \\
\hline 14 & & -.739 & & \\
\hline 16 & & -.716 & & \\
\hline 13 & .389 & -.606 & & \\
\hline 15 & & -.587 & & \\
\hline 35 & & -.452 & & \\
\hline 39 & & & .780 & \\
\hline 40 & & & .769 & \\
\hline 42 & & & .705 & \\
\hline 43 & & & .632 & \\
\hline 41 & & & .625 & \\
\hline 07 & & & .549 & \\
\hline 05 & .397 & & .538 & \\
\hline 44 & & & & .757 \\
\hline 47 & & & & .680 \\
\hline 45 & & & & .635 \\
\hline 46 & & & & .573 \\
\hline 49 & & & & .537 \\
\hline 36 & & & & .477 \\
\hline 06 & .305 & & & .417 \\
\hline 23 & & & & .398 \\
\hline
\end{tabular}

For ease of interpretation only loadings above .3 are displayed.

between directly comparable scales (e.g. cognitive to cognitive) were of the magnitude of 0.7 .

\section{Raw score to interval scale conversion}

Given fit to the Rasch model, Table 7 provides a simple conversion of the raw score for each scale, to its interval scale equivalent.

\section{Discussion}

Fatigue is an important symptom in many chronic diseases, and can have a considerable impact upon lifestyle $[37,38]$. Despite this, the scales used in the measurement of MS fatigue in health outcome studies have been shown to fall short of current standards, partly indicative of the lack of a clear definition of the construct $[11,12]$. Concern about the quality of existing measures led to a new study which, using qualitative approaches, introduced a detailed definition of fatigue and a scale with an original item set reflecting that definition [6].

No a priori assumptions regarding the dimensionality of fatigue were imposed for the derivation of the item subsets from the qualitative work. However, a fundamental requirement for unidimensionality is an assumption of the Rasch model and this, together with the exploratory factor analysis, guided the eventual subscales of the NFI-MS. In practice, the resulting domains were in accordance with the conceptual dimensions found in the qualitative phase, including the notion that the sub-dimensions were part of a single, supraordinate theme of 'neurological fatigue'.

Fit of scale data to the Rasch model also allows for a transformation of the ordinal raw score to an interval scale latent estimate which, given appropriate distributions, can be used in parametric procedures. There is a straightforward ordinal to interval scale equivalence, courtesy of a special property of the Rasch model called specific objectivity, [39] and this has been provided in the nomogram of Table 7 . This equivalence table is only valid provided there are no missing data in the raw scores of any new sample.

\section{Strengths and limitations}

In this study the Neurological Fatigue Index (NFI-MS) has been developed to meet the most rigorous, modern psychometric qualities for measurement. A combination of factor analysis and Rasch analysis led to strictly unidimensional scales for physical and cognitive fatigue, as well as a short summary scale. These solutions were validated upon a set-aside or validation sample and thus can be considered robust with respect to their internal construct validity. The magnitude of correlations between the physical and cognitive components and appropriate comparator measures also give support to the external construct validity of the scales.

Understanding of the full processes involved in fatigue is still in its infancy [40]. The production of a definition of fatigue and its measurement therefore might be in itself a worthy goal, but it was envisaged from the outset that these would just be the necessary first steps to exploration of the pathophysiology of the symptom. 
Table 4 Summary fit statistics for Rasch analyses

\begin{tabular}{|c|c|c|c|c|c|c|c|c|c|}
\hline \multirow{2}{*}{$\begin{array}{c}\text { Analysis Name } \\
\text { Evaluation Sample }\end{array}$} & \multicolumn{2}{|c|}{ Item Residual } & \multicolumn{2}{|c|}{ Person Residual } & \multicolumn{2}{|c|}{ Chi-Square } & \multirow[b]{2}{*}{ PSI } & \multirow{2}{*}{$\begin{array}{c}\text { Uni-dimensional } \\
\text { t-test }(\mathrm{Cl})\end{array}$} & \multirow[t]{2}{*}{$\%$ extreme scores in final versions } \\
\hline & Mean & $S D$ & Mean & $S D$ & Value & $p$ & & & \\
\hline 1. Physical set up & -0.02 & 2.353 & -0.264 & 1.385 & 172 & 0.056 & 0.946 & $\begin{array}{c}14.60 \% \\
(12.2-17.0)\end{array}$ & \\
\hline 2. Physical Final & 0.066 & 0.867 & -0.337 & 1.098 & 62.8 & 0.77 & 0.905 & $\begin{array}{l}6.03 \% \\
(3.6-8.4)\end{array}$ & $8.52 \%$ \\
\hline 3. Cognitive Set Up & -0.563 & 3.058 & -0.478 & 1.362 & 179.9 & $<0.001$ & 0.902 & $\begin{array}{l}6.71 \% \\
(4.3-9.1)\end{array}$ & \\
\hline 4. Cognitive Final & 0.21 & 0.623 & -0.432 & 1.041 & 24.3 & 0.665 & 0.849 & $4.46 \%$ & $11.00 \%$ \\
\hline 5. Diurnal sleep Set Up & -0.019 & 0.989 & -0.443 & 1.293 & 49.7 & 0.801 & 0.864 & $\begin{array}{c}5.75 \% \\
(3.3-8.2)\end{array}$ & \\
\hline 5a. Diurnal sleep modified & -0.069 & 1.136 & -0.451 & 1.235 & 68.9 & 0.083 & 0.845 & $4.95 \%$ & $3.78 \%$ \\
\hline 6. Nocturnal Sleep Set Up & 0.208 & 1.873 & -0.378 & 1.379 & 127.3 & $<0.001$ & 0.822 & $\begin{array}{c}5.7 \\
(3.3-8.2)\end{array}$ & \\
\hline 7. Nocturnal Sleep Final & 0.285 & 1.389 & -0.401 & 1.378 & 79.2 & 0.081 & 0.821 & $2.85 \%$ & \\
\hline 7a. Nocturnal Sleep modified & 0.26 & 1.466 & -0.399 & 1.209 & 56.4 & 0.118 & 0.761 & $2.95 \%$ & $3.47 \%$ \\
\hline 8. Summary Scale Set up & 0.06 & 2.319 & -0.332 & 1.631 & 370.4 & $<0.001$ & 0.936 & $\begin{array}{c}10.79 \% \\
(8.4-13.2)\end{array}$ & \\
\hline $\begin{array}{l}\text { 9. Summary scale Final } \\
\text { Validation Sample }\end{array}$ & -0.077 & 1.173 & -0.31 & 1.144 & 106.2 & 0.117 & 0.916 & $5.40 \%$ & $6.62 \%$ \\
\hline 10. Physical & 0.066 & 0.867 & -0.337 & 1.098 & 62.9 & 0.77 & 0.905 & $\begin{array}{c}6.03 \% \\
(3.6-8.4)\end{array}$ & $7.23 \%$ \\
\hline 11. Cognitive & 0.234 & 0.739 & -0.358 & 0.994 & 22.8 & 0.74 & 0.842 & $3.15 \%$ & $10.38 \%$ \\
\hline 12. Summary & 0.16 & 1.329 & -0.381 & 1.284 & 97.4 & 0.278 & 0.898 & $\begin{array}{l}6.62 \% \\
(4.2-9.0)\end{array}$ & $2.83 \%$ \\
\hline 13. Diurnal Sleep & 0.041 & 1.158 & -0.462 & 1.335 & 58.8 & 0.305 & 0.843 & $\begin{array}{c}7.69 \% \\
(5.3-10.1)\end{array}$ & \\
\hline 14. Diurnal Sleep Modified & 0.069 & 1.136 & 0.451 & 1.235 & 68.9 & 0.083 & 0.845 & $4.76 \%$ & $4.09 \%$ \\
\hline 15. Nocturnal Sleep & 0.235 & 1.817 & -0.377 & 1.293 & 102.1 & 0.001 & 0.808 & $\begin{array}{c}5.99 \% \\
(3.6-8.4)\end{array}$ & \\
\hline 16. Nocturnal Sleep modified & 0.26 & 1.466 & -0.399 & 1.209 & 56.4 & 0.118 & 0.761 & $3.15 \%$ & $3.77 \%$ \\
\hline Ideal Values & 0 & $<1.4$ & 0 & $<1.4$ & & $>0.05^{\mathrm{a}}$ & $>0.85$ & $<5.0 \%(C l)$ & \\
\hline
\end{tabular}

${ }^{a}$ Bonferroni adjusted alpha level

$\mathrm{PSI}=$ person separation index; $\mathrm{Cl}=$ confidence interval (only shown for values over $5 \%$ )

Thus the focus of this development has been upon the impairment of function as opposed to the social impact of fatigue. Nevertheless, the multi-dimensional nature of fatigue in MS lends itself to an exploration of the role of fatigue in the more complex bio-psychosocial model as expressed though the International Classification of Functioning, Disability and Health (ICF)[41].

Table 5 Test-retest comparisons

\begin{tabular}{ccc}
\hline Scale & Spearman rho* & $\begin{array}{c}\text { Median Scores } \\
\text { T1, T2 }\end{array}$ \\
\hline Summary & 0.864 & 21,20 \\
Physical & 0.852 & 17,16 \\
Cognitive & 0.826 & 7,6 \\
Nocturnal sleep & 0.837 & 8,8 \\
Diurnal sleep & 0.796 & 11,10 \\
\hline
\end{tabular}

Spearman correlation coefficients and median scores for subscales and Summary scores over 2-4 week period.

* all $\mathrm{p}<0.001$

$\mathrm{T} 1$ = initial completion, $\mathrm{T} 2$ = retest at $2-4$ weeks

** all differences, by Wilcoxon Signed Rank, non-significant $(p>0.05$ )
The use of factor analytical techniques on ordinal data, although widespread in psychology and health outcomes, nevertheless remains contentious [42,43]. We have attempted to overcome some of these limitations by using a polychoric correlation matrix as the basis of our exploratory analysis, and parallel analysis to determine significant eigenvalues, but have otherwise used the procedures available in SPSS which would be widely available. Our previous work on simulated multidimensional data has indicated that this is a reasonably robust approach for a simple exploration of factorial structures in polytomous data [33].

At the present time these data are only supportive of the validity of the scales within MS, and thus the instrument should be considered to be the NFI-MS. However, further work is underway to validate the item set in Stroke and MND. This may confirm the generic validity of the existing subscales, or it may be suggestive of alternative subscale structures. This is an empirical matter and, until further evidence is available, the label NFI-MS should be used. 
Table 6 External construct validity

\begin{tabular}{|c|c|c|c|c|c|}
\hline Scale & Summary & Physical & Cognitive & Nocturnal sleep & Diurnal sleep \\
\hline Physical & 0.96 & & & & \\
\hline Cognitive & 0.85 & 0.71 & & & \\
\hline Nocturnal sleep & 0.62 & 0.60 & 0.55 & & \\
\hline Diurnal sleep & 0.65 & 0.63 & 0.55 & 0.51 & \\
\hline MFIS phys-8 & 0.71 & 0.72 & 0.55 & 0.44 & 0.51 \\
\hline MFIS cog-5 & 0.58 & 0.48 & 0.69 & 0.46 & 0.37 \\
\hline FSS-5 & 0.71 & 0.71 & 0.57 & 0.43 & 0.54 \\
\hline VAS & 0.67 & 0.67 & 0.52 & 0.50 & 0.46 \\
\hline
\end{tabular}

Pearson correlation coefficients (Spearman for the VAS) between the Rasch derived person locations of the NFI-MS scales and the comparator scales. $\mathrm{p}<0.001$ for all correlations.

MFIS = Modified Fatigue Impact Scale, FSS = Fatigue Severity Scale, VAS = visual analogue scale.

Table 7 Raw score to interval scale conversion table

\begin{tabular}{|c|c|c|c|c|c|}
\hline Raw Score & $\begin{array}{l}\text { Summary } \\
\text { Scale }\end{array}$ & $\begin{array}{l}\text { Physical } \\
\text { Scale }\end{array}$ & $\begin{array}{c}\text { Diurnal Sleep } \\
\text { Scale }\end{array}$ & $\begin{array}{c}\text { Nocturnal Sleep } \\
\text { Scale }\end{array}$ & $\begin{array}{l}\text { Cognitive } \\
\text { Scale }\end{array}$ \\
\hline 0 & 0.00 & 0.00 & 0.00 & 0.00 & 0.00 \\
\hline 1 & 2.49 & 1.91 & 1.71 & 2.04 & 1.38 \\
\hline 2 & 4.26 & 3.33 & 3.03 & 3.53 & 2.58 \\
\hline 3 & 5.49 & 4.37 & 4.07 & 4.63 & 3.64 \\
\hline 4 & 6.48 & 5.24 & 4.97 & 5.55 & 4.62 \\
\hline 5 & 7.32 & 6.03 & 5.85 & 6.37 & 5.53 \\
\hline 6 & 8.07 & 6.75 & 6.72 & 7.12 & 6.36 \\
\hline 7 & 8.76 & 7.42 & 7.58 & 7.83 & 7.13 \\
\hline 8 & 9.42 & 8.09 & 8.46 & 8.52 & 7.89 \\
\hline 9 & 10.05 & 8.75 & 9.29 & 9.18 & 8.67 \\
\hline 10 & 10.65 & 9.42 & 10.09 & 9.85 & 9.54 \\
\hline 11 & 11.28 & 10.10 & 10.88 & 10.56 & 10.63 \\
\hline 12 & 11.91 & 10.81 & 11.63 & 11.31 & 12.00 \\
\hline 13 & 12.54 & 11.58 & 12.38 & 12.19 & \\
\hline 14 & 13.20 & 12.38 & 13.16 & 13.38 & \\
\hline 15 & 13.86 & 13.23 & 14.01 & 15.00 & \\
\hline 16 & 14.55 & 14.14 & 14.99 & & \\
\hline 17 & 15.30 & 15.06 & 16.27 & & \\
\hline 18 & 16.05 & 15.99 & 18.00 & & \\
\hline 19 & 16.83 & 16.95 & & & \\
\hline 20 & 17.64 & 17.93 & & & \\
\hline 21 & 18.45 & 18.97 & & & \\
\hline 22 & 19.29 & 20.22 & & & \\
\hline 23 & 20.13 & 21.85 & & & \\
\hline 24 & 21.03 & 24.00 & & & \\
\hline 25 & 21.96 & & & & \\
\hline 26 & 22.98 & & & & \\
\hline 27 & 24.12 & & & & \\
\hline 28 & 25.53 & & & & \\
\hline 29 & 27.42 & & & & \\
\hline 30 & 30.00 & & & & \\
\hline
\end{tabular}

The conversions remain valid provided there are no missing data. 


\section{Future directions}

Other future work could include the determination of imaging correlates and comparison of neurological fatigue experienced in MS and other diseases of the nervous system. This would be contingent upon the above validation studies in other conditions. Further validation of the sleep scales is also required, as these may form an important component of a bio-psychosocial model analysis. An understanding of the potential integral or adaptive roles of day and night sleep would be a high priority. Appropriate cross-cultural validation would allow the use of the NFI-MS as an outcome measure in internationally based clinical trials [28].

\section{Conclusion}

The NFI-MS provides a brief and easy-to-use tool for the measurement of fatigue in MS. It was developed from the reported experience of fatigue by patients in accordance with the latest FDA guidelines for scale development. A short summary scale is available, but underlying components can also be measured. Fit to the Rasch measurement model was rigorously tested and was found to be reproducible. Such fit means that interval level scaling is available when change scores need to be calculated. The scales have specific validation for MS and can be used on patients of any age, sex, and duration.

\section{Implications for practice and research}

It is suggested that the Summary scale would be useful in both a clinical setting and as an outcome measure in clinical trials and the different subscales would be suited to physiological and bio-psychosocial studies. Given fit to the Rasch model, the raw score is a sufficient statistic for identifying the (ordinal) level of fatigue in patients by simply adding up the raw score for the scale, which lends itself to convenient everyday use in a clinical setting. The ordinal-interval transformation could be used whenever parametric statistics are required. The NFIMS is free for use in all Public Health and not-for-profit agencies, and can be obtained from the authors following a simple registration.

\section{Acknowledgements}

The authors would like to thank: all the interviewees and respondents for their willingness in taking part in this study; Dr Richard Nicholas and Dr Omar Malik, of Imperial College Healthcare Trust, for allowing the approach of patients under their care; and Dave Watling and the staff of the Clinical Trials Unit, WCNN for their assistance with the mailout.

\section{Author details}

${ }^{1}$ The Walton Centre for Neurology and Neurosurgery, Liverpool, L9 7LJ, UK. ${ }^{2}$ School of Rural Health, University of Melbourne, 49 Graham St, Shepparton, Victoria, 3630, Australia. ${ }^{3}$ Department of Rehabilitation Medicine, Faculty of Medicine and Health, University of Leeds, D Floor, Martin Wing, Leeds General Infirmary, Gt George Street, Leeds, LS1 3EX, UK.

\section{Authors' contributions}

RJM and CAY contributed to the design, implementation, and analysis of the study. JFP and AT contributed to the analysis of the study. All authors contributed to the writing of the manuscript, and all approved the final version.

\section{Competing interests}

The authors declare that they have no competing interests.

Received: 12 November 2009

Accepted: 12 February 2010 Published: 12 February 2010

\section{References}

1. Freal JE, Kraft GH, Coryell JK: Symptomatic fatigue in multiple sclerosis. Arch Phys Med Rehabil 1984, 65(3):135-138.

2. Comi $G$, Leocani $L$, Rossi $P$, Colombo B: Physiopathology and treatment of fatigue in multiple sclerosis. J Neurol 2001, 248(3):174-179.

3. Johansson S, Ytterberg C, Hillert J, Widen Holmqvist L, von Koch L: A longitudinal study of variations in and predictors of fatigue in multiple sclerosis. J Neurol Neurosurg Psychiatry 2008, 79(4):454-457.

4. Krupp LB, LaRocca NG, Muir-Nash J, Steinberg AD: The fatigue severity scale. Application to patients with multiple sclerosis and systemic lupus erythematosus. Arch Neurol 1989, 46(10):1121-1123.

5. Multiple Sclerosis Council: Fatigue and Multiple Sclerosis-Clinical Practice Guidelines. Washington D.C.: Paralyzed Veterans of America 1998

6. Mills RJ, Young CA: A medical definition of fatigue in multiple sclerosis. QJM 2008, 101(1):49-60.

7. Krupp LB, Alvarez LA, LaRocca NG, Scheinberg LC: Fatigue in multiple sclerosis. Arch Neurol 1988, 45(4):435-437.

8. Kos D, Kerckhofs E, Nagels G, D'Hooghe MB, Ilsbroukx S: Origin of fatigue in multiple sclerosis: review of the literature. Neurorehabil Neural Repair 2008, 22(1):91-100.

9. Fisk JD, Doble SE: Construction and validation of a fatigue impact scale for daily administration (D-FIS). Qual Life Res 2002, 11(3):263-272.

10. Fischer JS, LaRocca NG, Miller DM, Ritvo PG, Andrews H, Paty D: Recent developments in the assessment of quality of life in multiple sclerosis (MS). Mult Scler 1999, 5(4):251-259.

11. Mills R, Young C, Nicholas R, Pallant J, Tennant A: Rasch analysis of the Fatigue Severity Scale in multiple sclerosis. Mult Scler 2009, 15(1):81-87.

12. Mills RJ, Young CA, Pallant J, Tennant A: Rasch analysis of the Modified Fatigue Imapct Scale (MFIS) in multiple sclerosis. JNNP 2009.

13. Rasch G: Probabilistic Models for Some Intelligence and Attainment Tests. Chicago: Univ Chicago P 1980.

14. US Food and Drug Administration: Draft guidance for industry on patientreported outcome measures: use in medicinal product development to support labeling claims (Docket 2006D-0044). Fed Register 2006, 71:5862-5863.

15. Likert RA: A technique for the development of attitude scales. Educat Psychol Measurement 1952, 12:313-315.

16. Polman $\mathrm{CH}$, Reingold SC, Edan G, Filippi M, Hartung HP, Kappos L, Lublin FD, Metz LM, McFarland HF, O'Connor PW, Sandberg-Wollheim M, Thompson AJ, Weinshenker BG, Wolinsky JS: Diagnostic criteria for multiple sclerosis: 2005 revisions to the "McDonald Criteria". Ann Neurol 2005, 58(6):840-846.

17. Kurtzke JF: Rating neurologic impairment in multiple sclerosis: an expanded disability status scale (EDSS). Neurology 1983, 33(11):1444-1452.

18. Bartlett MS: A note on multiplying factors for various chi square approximations. Journal of the Royal Statistical Society 1954, 16(Series B):296-298.

19. Kaiser HF: A second-generation Little Jiffy. Psychometrika 1970, 35:401-415.

20. Kaiser HF: An index of factorial simplicity. Psychometrika 1974, 39:31-36.

21. Kaiser HF: The application of electronic computers to factor analysis. Educational and Psychological Measurement 1960, 20:141.

22. Cattell RB: The scree test for the number of factors. Multivariate Behavioral Research 1966, 1:245-276.

23. Horn JL: A Rationale and Test for the Number of Factors in Factor Analysis. Psychometrika 1965, 30:179-185.

24. Hubbard R, Allen S: An empirical comparison of alternative methods for principal component extraction. J Bus Res 1987, 15:173-190.

25. Watkins M: Monte Carlo PCA for Parallel Analysis. State College, PA: Ed and Psych Associates 2000 
26. Conrad KJ, Wright BD, McKnight P, McFall M, Fontana A, Rosenheck R: Comparing traditional and Rasch analyses of the Mississippi PTSD Scale: revealing limitations of reverse-scored items. J Appl Meas 2004, 5(1):15-30.

27. Mills RJ, Young CA, Woolmore JA, Hawkins CP: A final UK scale for measurement of self efficacy in MS. Mult Scler 2006, 12(S1):S91.

28. Kucukdeveci AA, Sahin H, Ataman S, Griffiths B, Tennant A: Issues in crosscultural validity: example from the adaptation, reliability, and validity testing of a Turkish version of the Stanford Health Assessment Questionnaire. Arthritis Rheum 2004, 51(1):14-19.

29. Pallant JF, Tennant A: An introduction to the Rasch measurement model: an example using the Hospital Anxiety and Depression Scale (HADS). $\mathrm{Br}$ J Clin Psychol 2007, 46(Pt 1):1-18

30. Tennant A, Conaghan PG: The Rasch measurement model in rheumatology: what is it and why use it? When should it be applied, and what should one look for in a Rasch paper?. Arthritis Rheum 2007 57(8):1358-1362

31. Tennant A, Pallant J: DIF matters. Rasch Measurement Transactions 2006, 20:1082-1084.

32. Smith EV Jr: Detecting and evaluating the impact of multidimensionality using item fit statistics and principal component analysis of residuals. J Appl Meas 2002, 3(2):205-231.

33. Tennant A, Pallant JF: Unidimensionality Matters! (A Tale of Two Smiths?). Rasch Measurement Transactions 2006, 20(1):1048-1051.

34. Choppin B: A fully conditional estimation procedure for Rasch model parameters (CSE report 196). University of California, Center for the Study of Evaluation 1983

35. Linacre JM: Sample size and item calibration stability. Rasch Measurement Transactions 1994, 7:28

36. Andrich D, Lyne A, Sheridan B, Luo G: RUMM 2020. Perth, Australia: RUMM Laboratory Pty. Ltd 2007

37. MCElhiney MC, Rabkin JG, Gordon PH, Goetz R, Mitsumoto H: Prevalence of fatigue and depression in ALS patients and change over time. J Neurol Neurosurg Psychiatry 2009, 80(10):1146-1149.

38. Wolfe F, Michaud K: Predicting depression in rheumatoid arthritis: the signal importance of pain extent and fatigue, and comorbidity. Arthritis Rheum 2009, 61(5):667-673.

39. Rasch G: On general laws and the meaning of measurement in psychology. Proceedings of the Fourth Berkeley Symposium on Mathematical Statistics and Probability 1961, 4:321-333.

40. Trojan DA, Arnold D, Collet JP, Shapiro S, Bar-Or A, Robinson A, Le Cruguel JP, Ducruet T, Narayanan S, Arcelin K, Wong AN, Tartaglia MC, Lapierre Y, Caramanos Z, Da Costa D: Fatigue in multiple sclerosis: association with disease-related, behavioural and psychosocial factors. Mult Scler 2007, 13(8):985-995.

41. World Health Organization: International classification of functioning disability and health: ICF. Geneva: WHO 2001

42. Gilley WF, Uhlig GE: Factor Analysis and Ordinal Data. Education 1993 114(2):258-264.

43. Joreskog K, Moustaki I: Factor Analysis for Ordinal Variables: a Comparison of three approaches. Multivariate Behavioural Research 2001, 36:347-387.

doi:10.1186/1477-7525-8-22

Cite this article as: Mills et al:: Development of a patient reported outcome scale for fatigue in multiple sclerosis:

The Neurological Fatigue Index (NFI-MS). Health and Quality of Life Outcomes 2010 8:22.

\section{Submit your next manuscript to BioMed Central and take full advantage of:}

- Convenient online submission

- Thorough peer review

- No space constraints or color figure charges

- Immediate publication on acceptance

- Inclusion in PubMed, CAS, Scopus and Google Scholar

- Research which is freely available for redistribution

Submit your manuscript at www.biomedcentral.com/submit 